\title{
RECALCITRANT PRURITUS AS PRIMARY MANIFESTATION OF SYNCHRONOUS HODGKINS LYMPHOMA AND LANGERHANS CELL HISTIOCYTOSIS
}

\author{
Afsha A. Topal', Ram H. Malkani', Ganapathi Bhat ${ }^{2}$, Reshma T. Vishnani ${ }^{1}$
}

Department of Dermatology ${ }^{1}$ and Department of Oncology ${ }^{2}$ Jaslok Hospital and Research Centre, Mumbai, India

Summary: We present a 73 year old female with intractable pruritus and nonspecific cutaneous rash for a period of 9 months. She had recieved symptomatic therapy with no improvement. A complete examination revealed axillary and abdominal lymphadenopathy. A biopsy confirmed the diagnosis of Hodgkins lymphoma with Langerhans cell histiocytosis. She received 5 cycles of chemotherapy with resolution of pruritus and reduction in axillary and abdominal lymphadenopathy. The patient presented 6 months later with relapse and succumbed to the illness. Simultaneous occurrence of Langerhans cell histiocytosis and Hodgkins lymphoma may lead to misdiagnosis. The awareness of such an association is important to make an accurate diagnosis and guide appropriate therapy.

Key words: Pruritus; Hodgkins lymphoma; Langerhans cell histiocytosis

\section{Case Report}

A 73 year old lady presented with generalised pruritic rash since a duration of 9 months. She had recieved various topical and systemic symptom oriented medications with no significant benefit. She had experienced weight loss of around $15 \mathrm{kgs}$ in the last 6 months and low grade fever since 1 month. Cutaneous examination revealed multiple erythematous excoriated papules, few showing scaling, over extremities (extensor > flexor), chest, abdomen and back (Fig. 1 and 2). Scalp and flexures were spared. General examination revealed firm, discrete and nontender left axillary lymphadenopathy. Initial investigations revealed anaemia (Hb: 10.4), a normal complete blood count and hypoproteinemia (Serum Total Protein $-6.7 \mathrm{~g} / \mathrm{dl}$, Serum Albumin - $3.4 \mathrm{~g} / \mathrm{dl}$ ). A skin biopsy from an erythematous papule showed spongiotic epidermis, a moderate superficial and deep perivascular infiltrate composed of lymphocytes, monocytes and several eosinophils, with no evidence of Langerhans cell histiocytosis or Hodgkins lymphoma (Fig. 3). Ultrasound of abdomen showed multiple hypoechoic retroperitoneal lymphnodes and multiple hypoechoic splenic deposits suggestive of a possibility of lymphoma. Positron emission tomography (PET) scan revealed evidence of high grade active disease in bilateral axillary, mediastinal, perihepatic, periportal, retroperitoneal, and bilateral iliac group of lymphnodes, involvement of the spleen, liver, left ischial and sacral bones (Fig. 4). Bone marrow biopsy (posterior iliac crest) was normal. Axillary lymph node biopsy was reported as Langerhans cell histiocytosis with expression of S100 and CD 1a. But, due to the strong clinical suspicion, the biopsy specimen was resectioned and reviewed by the same histopathologist. It showed CD30 positive Reed-Sternberg cells which were negative for CD 15, LCA, CD 20 and CD 3 (Fig. 5). On the basis of these findings the classification the patient was diagnosed as Classical Hodgkins lymphona, Stage IV according to the Ann Arbor classification and treated with pulsed injectable methylprednisolone followed by chemotherapy. She was given 1 cycle of CEPP (cyclo-phosphamide, etoposide, procarbazine, and prednisone) followed by 5 cycles of COPP (cyclophosphamide, vincristine, procarbazine, and prednisone), after which she achieved complete remission. There was resolution of pruritus and reduction in axillary and abdominal lymphadenopathy on abdominal ultrasound. A repeat PET scan did not reveal any disease activity. The patient

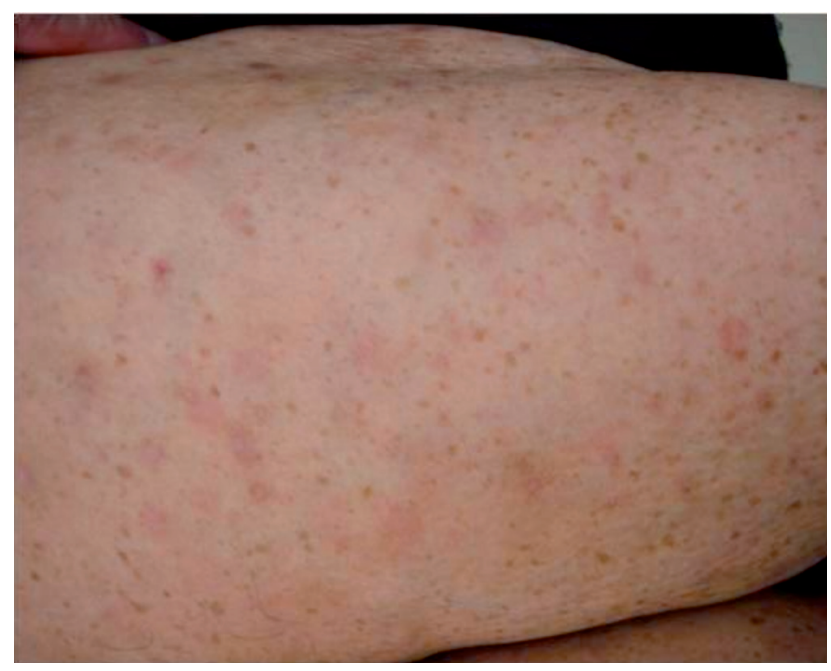

Fig. 1: Multiple erythematous papules, few excoriated over the thigh 


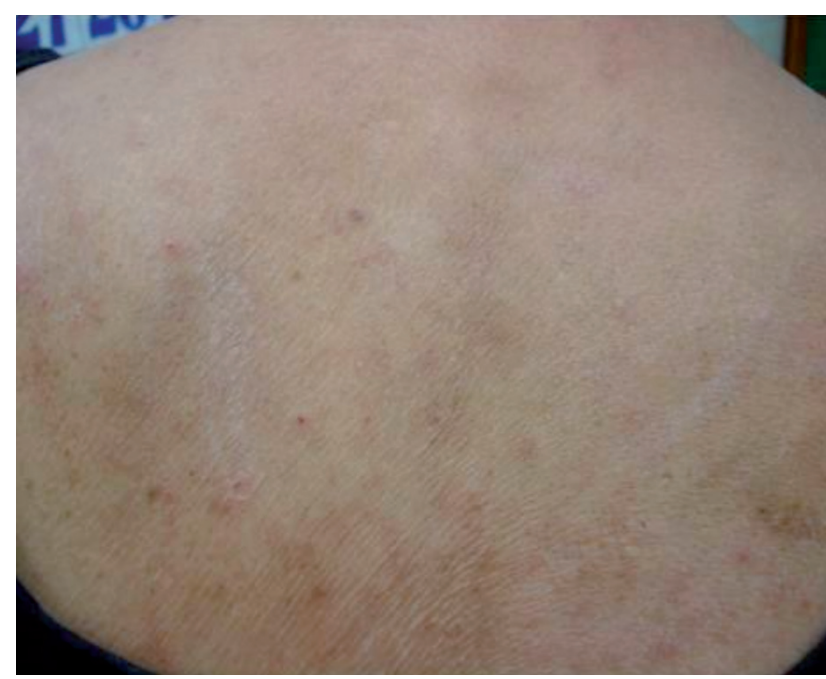

Fig. 2: Few excoriated papules with underlying erythema over the upper back

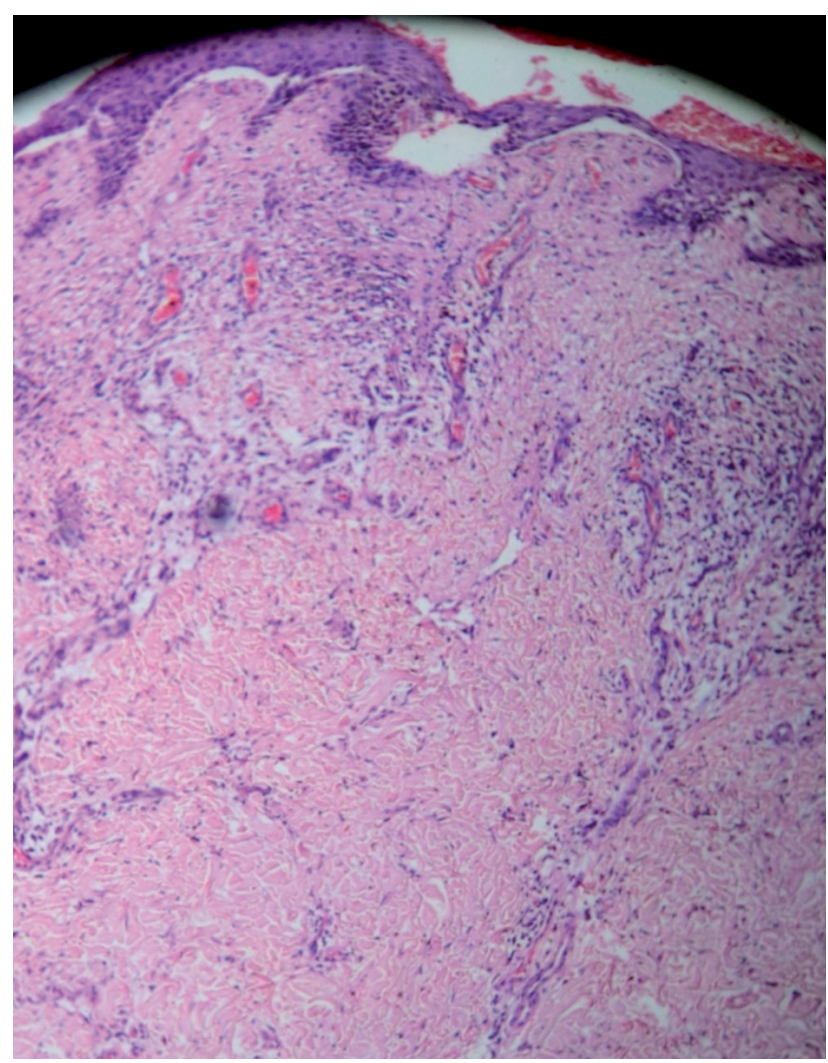

Fig. 3: A skin biopsy from an eythematous papule showed spongiotic epidermis, a moderate superficial and deep perivascular infiltrate composed of lymphocytes, monocytes and several eosinophils

presented 6 months later with history of itching and lethargy since 3 weeks. USG Abdomen showed multiple hypoechoic

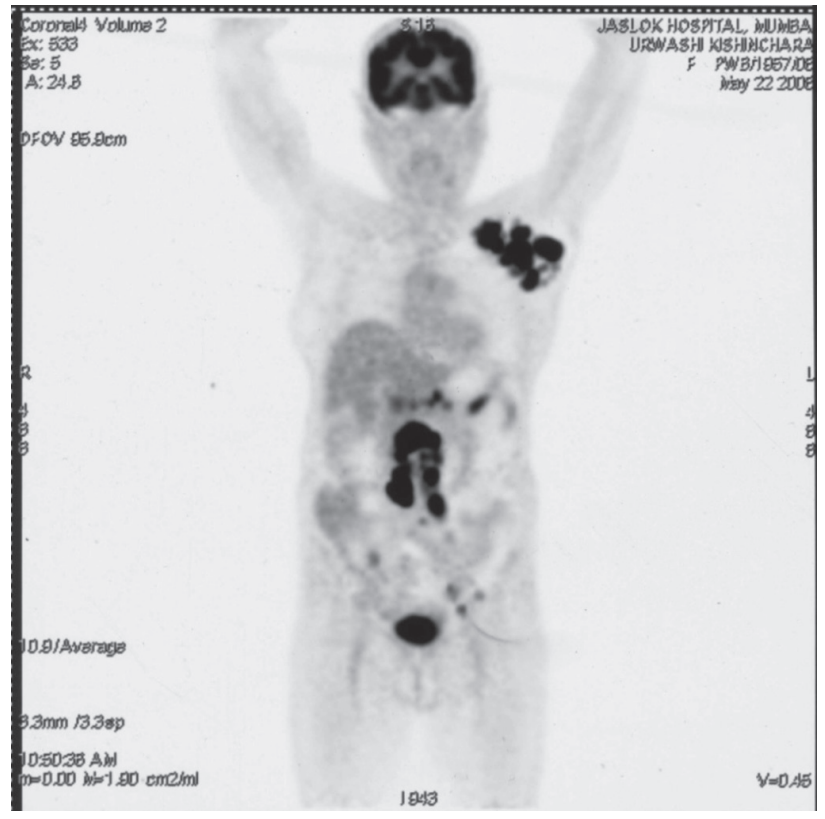

Fig. 4: Positron emission tomography (PET scan) revealed evidence of high grade active disease in bilateral axillary, mediastinal, perihepatic, periportal, retroperitoneal, and bilateral iliac group of lymphnodes

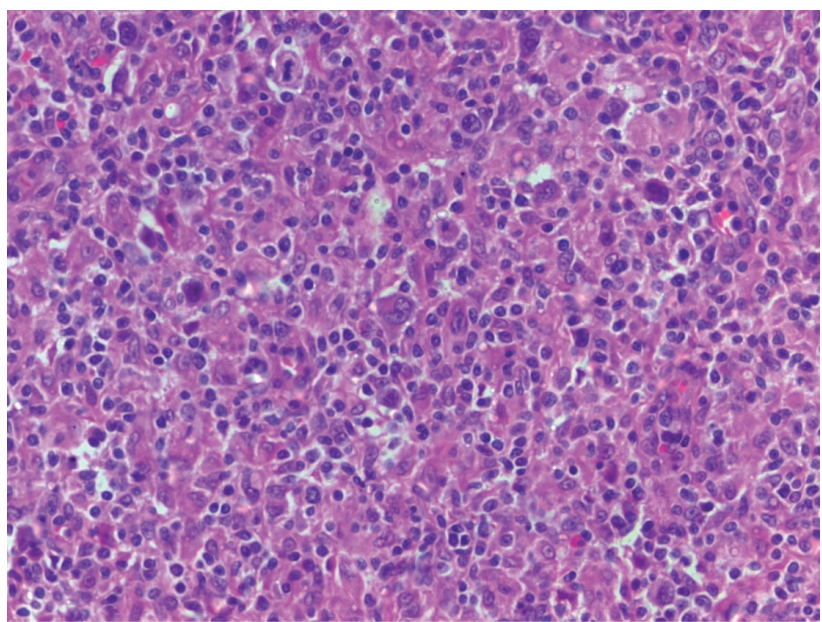

Fig. 5: Left axillary lymph node biopsy showed Reed-Sternberg cells suggestive of classical Hodgkins disease

lesion in liver and spleen, multiple lymphnodes seen at porta and in suprapancreatic region. Repeat PET scan showed evidence of high grade active disease in left axillary lymphnode, mediastinal, retroperitoneal lymphnodes and involvement of the spleen and liver. The patient was treated in relapse with 3 cycles of etoposide, procarbazine and prednisone and started on Tab. Chlorambucil $2 \mathrm{mg}$ daily. Repeat USG Abdomen showed multiple hypoechoic lesions in liver. Eventually, the patient developed septicaemia and succumbed to the disease. 


\section{Discussion}

Hodgkin's disease (HD) is rare in the elderly. In population-based studies, the proportion of HD patients over age 60 years has ranged from $15 \%$ to $30 \%$ (1). Several studies have shown that Hodgkin's disease in older adults has a poorer prognosis than in younger adults (2). Walker et al reanalysed data from the American College of Surgeons Patterns of Care Study (Kennedy et al, 1985) and found that advanced age remained an independent predictor of poor prognosis, even after correction for histological subtype, stage of disease, B symptoms and treatment (3). The most established prognostic tool in HD is the International Prognostic Score (IPS), which uses 7 adverse clinical prognostic factors, including age older than 45 years, to predict outcome (4). Epstein-Barr virus has been suspected as an aetiological agent in the pathogenesis of Hodgkin's disease for some years, however the relationship between EBV-associated (EBV-positive) Hodgkin's disease and prognosis is controversial. Studies have demonstrated an association between EBV positivity and a poor prognosis of Hodgkin's disease in older patients (5). Elderly individuals are recognized to have lower immunocompetence than younger people and may therefore have more difficulty in controlling the growth of an EBV-associated tumour than younger patients.

Generalized pruritus is noted in approximately $30 \%$ cases of Hodgkins disease (6) and maybe the only presenting symptom (7). Smith et al and White et al found that specific skin involvement in Hodgkins disease is rare, occurring in $0.5 \%$ and $3.4 \%$ respectively $(8,9)$. Nonspecific cutaneous symptoms maybe seen in upto $50 \%$ of patients (10) and include eczema (11), hyperpigmentation, prurigo, icthyosis, vasculitis, bullous eruption, erythema nodosum and generalized hyperhidrosis (12). Gobbi et al proposed inclusion of severe pruritus among Ann Arbor criteria for definition of the B-clinical category due to the intrinsically poor prognosis related to severe pruritus (6). But it was retrospective analysis from 1971-1979 years and pruritus was not added to B symptoms.

Langerhans cell histiocytosis has been described in association with a variety of other tumor types, preceding, after or synchronous with the other tumor. The most common associations are with malignant lymphomas and leukaemias (13). Most commonly, the diagnoses are concurrent, with the lymphoma being of Hodgkin type. This association is rare, and has been estimated to be $0.3 \%$ for Hodgkin disease (14). Shin et al discussed the relationship between these two conditions and proposed various possibilities (15):

1. Hodgkins disease induces Langerhans cell histiocytosis;

2. Radiotherapy and/or chemotherapy for Hodgkins disease leads to the development of Langerhans cell histiocytosis;
3. Langerhans cell histiocytosis may represent a specific cell mediated immune response to Hodgkins disease;

4. A common etiological agent induces both Hodgkins disease and Langerhans cell histiocytosis.

In concurrent occurrence of these two diseases, Langerhans cells are smaller than regular ones in histopathologic examination and this makes the differential diagnosis more difficult (16). PET imaging is not of much help in differentiating these two conditions, since both conditions show increase uptake of glucose (17). Hence, histopathology and immunohistochemistry are essential to distinguish these two conditions.

In our case, the presence of the characteristic pruritus, which is strongly suggestive of Hodgkins disease and the knowledge of the synchronous occurrence of these two conditions helped us reach the correct diagnosis. An accurate diagnosis in such cases guides the physician in the choice of therapy (conservative in Langerhans cell histiocytosis versus aggressive in Hodgkins disease).

\section{References}

1. Evens AM, Sweetenham JW, Horning SJ. Hodgkin lymphoma in older patients: an uncommon disease in need of study. Oncology 2008; 22(12): 1369-1379.

2. Stark GL, Wood KM, Jack F, Angus B, Proctor SJ, Taylor PR. On behalf of the Northern Region Lymphoma Group Hodgkin's disease in the elderly: a population-based study. British Journal of Haematology 2002; 119: 432-440.

3. Walker A, Schonfeld ER, Lowman JT et al. Survival of the older patient compared with the younger patient with Hodgkins disease. Cancer 1990; 65: 1635-1640.

4. Hasenclever D, Diehl V. A numerical index to predict tumor control in advanced Hodgkin's disease. Blood 1996 (suppl. 1, abstr.); 88: 673a.

5. Thorley-Lawson DA, Gross A. Persistence of the Epstein-Barr virus and the origins of associated lymphomas. N Engl J Med 2004; 350: 1328-1337.

6. Gobbi PG, Attardo Parinello G, Lattanzio G Rizzo SC, Ascari E. Severe pruritus should be a B symptom in hodgkins disease. Cancer 1983; 51: 1934-6.

7. O'Donell BF, Alton B, Carnney D, Loughlin S. Generalized pruritus when to investigate further. J Am Acad Dermatol 1993; 28: 117

8. Smith JL Jr, Butler JJ. Skin involvement in hodgkins disease cancer 1980; 45: 354-61.

9. White RM, Patterson JW. Cutaneous involvement in hodgkins disease. Cancer 1985; 55: 1136-1145.

10. Szur L, Harrison CV, Levene GM, Samman PD. Primary cutaneous hodgkins disease Lancet 1970; 1: 1016-20.

11. Callen JP, Bernadi DM, Clark RA, Weber DA. Adult onset recalcitrant eczema: marker of noncutaneous lymphoma or leukaemia J Am Acad Dermatol 2000; 43: 207-10.

12. Rubenstein M, Duvic M. Cutaneous manifestations of hodgkins disease. Int J Dermatol 2006; 45: 251-6.

13. Egeler RM, Neglia JP, Arico M et al. The relation of langerhans cell histiocytosis to acute leukaemia, lymhomas and other solid tumors. Hematol Oncol Clin North Am 1998; 12: 369-78.

14. Barnes BF, Colby TV, Dorfman RF. Langerhans cell granulomatosis associated with malignant lymphomas. Am J Surg Pathol. 1983; 7: 529-533.

15. Myung S. Shin, Scott E. Buchalter, Kang-Jey Ho. Langerhans' cell histiocytosis associated with Hodgkin's disease: A case report. J NatI Med Assoc. 1994; 86: 65-69.

16. Adu-Poku K, Thomas DW, Khan MK, Holgate CS, Smith MEF. Langerhans cell histiocytosis in sequential discordant lymphoma. J Clin Pathol 2005; 58(1): 104-6.

17. Naumann R, Beuthein Baumann B, Fischer R, Kittner T, Bredow J, Kropp J et al. Simultaneous occurrence of Hodgkins lymphoma and eosinophilic granuloma, a potential pitfall in positron emission tomography imaging. Clin lymphoma 2002; 3(2): 121-4.

Received: $16 / 01 / 2012$

Accepted in revised form: 09/06/2012

\section{Corresponding author:}

Dr. Afsha A. Topal, OPD 200, 2nd Floor, Department of Dermatology, Jaslok Hospital and Research Centre, 15, Dr. G. Deshmukh Marg, Mumbai-400 026, India; e-mail: afshatopal_11@yahoo.co.in 